\title{
PANORAMA DA ELETROQUÍMICA E ELETROANALÍTICA NO BRASIL
}

Maria Valnice Boldrin Zanoni ${ }^{\mathrm{a}, *}$, Alessandro Santos Borges ${ }^{\mathrm{b}}$, Assis Vicente Benedettia, Hideko Yamanaka ${ }^{\mathrm{a}}$, Maria del Pilar

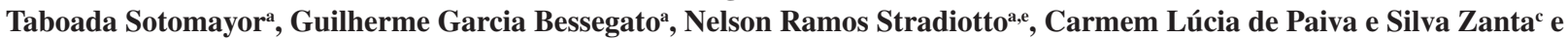
Adalgisa Rodrigues de Andrade ${ }^{d}$

Instituto de Química, Universidade Estadual Paulista, 14800-060 Araraquara - SP, Brasil

'Pró-Reitoria de Pesquisa, Universidade Estadual Paulista, 01049-010 São Paulo - SP, Brasil

'Instituto de Química e Biotecnologia, Universidade Federal de Alagoas, 57072-970 Maceió - AL, Brasil

¿Departamento de Química, Faculdade de Filosofia, Ciências e Letras de Ribeirão Preto, Universidade de São Paulo, 14040-901

Ribeirão Preto - SP, Brasil

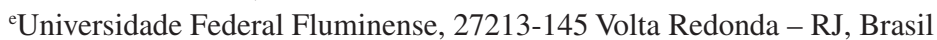

Recebido em 31/03/2017; aceito em 22/05/2017

\begin{abstract}
OVERVIEW OF THE ELECTROCHEMISTRY AND ELECTROANALYTICAL CHEMISTRY IN BRAZIL. Electrochemistry and Electroanalytical chemistry play an important role to face the challenges of the contemporary world. They have contributed to the advance of the scientific development of our country, helped to promote economic and social progress as well as cultural dissemination. These areas have greatly contributed to the knowledge about thermodynamics and kinetics of the electron transfer reactions, energy conversion, synthesis and modification of compounds, corrosion, and development of fast, sensitive, and selective analytical methods. This work presents an overview of the areas by analyzing the technical and scientific papers produced in Brazil on these subjects, and the analysis of the Brazilian scientific societies that were formed and consolidated, which have contributed to spread the scientific knowledge. The improvement of these areas can be attributed to the good quality of the human resources formed during long time as well as the growing of distribution of research groups in different regions of the country. The success/ future of these areas are associated with good academic/scientific education, low cost of instrumentation, formation of networks of excellence between researchers, and large cooperation projects that improved with the sharing of ideas, technologies, infrastructure, and equipment. All of which has contributed to strengthening consolidated and emerging graduation programs.
\end{abstract}

Keywords: Trends in Electrochemistry; Electroanalytical chemistry assessment; research in Electrochemistry and Electroanalysis.

\section{INTRODUÇÃO}

A Eletroquímica se caracteriza pela natureza universal e multidisciplinar e desempenha papel importante no desenvolvimento da ciência, com reflexos no crescimento econômico e melhoramento da qualidade de vida. Pode ser definida de modo mais abrangente como o ramo da Química que lida com reações químicas que envolvem corrente elétrica e potencial. Algumas reações químicas que ocorrem espontaneamente podem gerar corrente elétrica, que pode ser usada para fazer trabalho útil; enquanto outras reações químicas podem ser forçadas a prosseguir usando corrente elétrica.

A história da Eletroquímica começa no século XVIII quando Luigi Galvani (1737-1798) estabeleceu uma ponte entre contração muscular e eletricidade, e Alessandro Volta (1745-1827) anunciou a invenção da pilha (ou célula) voltaica, a primeira bateria elétrica moderna.

Enquanto seus antecessores, incluindo Benjamin Franklin (17061790), contribuíram para o entendimento da eletricidade estática, a pilha voltaica produzia corrente contínua e assim abriu duas novas áreas de estudo: a produção química de eletricidade e os efeitos da eletricidade sobre os compostos químicos. As descobertas de Michael Faraday (1791-1867) que resultaram nas duas leis fundamentais da Eletroquímica, publicadas em 1834, predizem quanto produto resulta ao passar uma certa quantidade de carga através de um composto químico ou de sua solução, processo que ele chamou de "eletrólise". Essas leis são a base para muitas técnicas eletroquímicas e eletroanalíticas. Ainda pode-se ressaltar a constatação de William Nicholson

*e-mail: boldrinv@iq.unesp.br
(1735-1815) e Anthony Carlisle (1768-1840) de que hidrogênio e oxigênio eram produtos da eletrólise da água.

A Eletroquímica tem desempenhado papel importante na resposta aos desafios do mundo contemporâneo. Sua grande contribuição no desenvolvimento de novos materiais, novas formas de energia, computadores, técnicas espectroscópicas para estudos de superfície etc., tem resultado em grandes avanços no mundo cotidiano na área de baterias, células solares, células a combustível, produção de hidrogênio, cloro, soda, alumínio, cobre, zinco, prata, chumbo e outros materiais que têm revolucionado a produção de joias, o desempenho dos carros, corrosão etc. Além das contribuições ao estudo datermodinâmica e cinética de reações, conversão de energia, síntese e modificação de compostos químicos, ainda tem demonstrado grande aplicabilidade no desenvolvimento de novos métodos analíticos.

A Eletroanalítica, também conhecida como Eletroanálise, está na interface entre ciência analítica e eletroquímica. Trata-se do desenvolvimento, caracterização, entendimento e aplicação de métodos de análise química empregando fenômenos eletroquímicos. Neste contexto, teve seu grande reconhecimento em 1922 quando Jaroslav Heyrovský (1890-1967) desenvolveu a técnica de polarografia usando um eletrodo gotejador de mercúrio capaz de monitorar reações eletroquímicas envolvendo metais, compostos orgânicos e organometálicos. Em 1959 teve seu trabalho reconhecido pela grande contribuição na área da Eletroanalítica e foi laureado com o prêmio Nobel. Em 1975, Royce W. Murray introduziu um novo enfoque na Eletroanalítica quando propôs que a imobilização ou incorporação de grupos químicos específicos ou microestruturas na superfície ou no corpo do eletrodo de trabalho pode gerar eletrodos modificados 
com maior aplicabilidade analítica por melhorarem a detectabilidade, a seletividade e interação analito/eletrodo. Estes dispositivos têm contribuído para o entendimento de vários processos eletroquímicos (catálise, inibição e transferência de carga), fotoeletroquímicos e determinações analíticas.

$\mathrm{Na}$ ciência analítica moderna, a Eletroanalítica tem papel importante no desenvolvimento de novos métodos de análise, sensores químicos, dispositivos e sistemas capazes de identificar, monitorar e quantificar os mais diversos tipos de analitos nas mais diferentes matrizes, tais como fluídos biológicos, matrizes ambientais, microssistemas e outros universos que requerem avanços tecnológicos para resoluções de problemas cada vez mais complexos.

Assim, desde os primórdios da Eletroquímica até o momento atual tem-se observado um considerável crescimento, tanto em magnitude quanto em diversificação. No Brasil, isto tem se tornado evidente pelo elevado número de trabalhos publicados na literatura técnica e científica, pela formação e consolidação de sociedades científicas profissionais e diferentes subdivisões, por uma série de congressos que reúnem pesquisadores nacionais e internacionais que ocorrem regularmente e, principalmente, pela notória qualidade na formação de recursos humanos na área. A seguir será dado uma visão global da evolução da Eletroquímica e da Eletroanalítica como áreas de vanguarda na química brasileira.

\section{A PRODUÇÃO CIENTÍFICA DA ELETROQUÍMICA E ELETROANALÍTICA NO BRASIL}

Uma avaliação crítica dos indicadores de produção acadêmico/ científico em Eletroquímica/ Eletroanalítica (EE) no mundo e a contribuição do Brasil neste contexto foi obtida pela busca de publicações na base de dados "InCites" (base da Web of Science da Thomson Reuters). Esta é uma ferramenta online de avaliação de pesquisa personalizada que compila dados bibliográficos e de citações da Web of Science multidisciplinar de mais de 100 países. Devido à grande sobreposição das pesquisas realizadas em Eletroquímica e Eletroanalítica, as publicações não puderam ser individualizadas e os dados obtidos consideraram a Eletroquímica em geral publicada em 208 periódicos.
A publicação científica nas áreas de EE aumentou exponencialmente nos últimos 10 anos (período mais significativo em relação ao crescimento da área), alcançando uma produção de 111.970 artigos no período de 2006-2015, o que representa 5\% da produção global em química. A Figura 1 ilustra a evolução da publicação científica na área de EE neste período e compara o Brasil com alguns países com liderança científica na área.

Na comparação da produção científica mundial destacam-se a produção científica da China, Estados Unidos da América (EUA), Japão, Coréia do Sul e Alemanha. Dentre eles, nos últimos anos, destaca-se a supremacia da China e EUA. No entanto, embora em 2006 a produção da China era de $17 \%$ menor em relação aos EUA, em 2015 sua produção científica é $219 \%$ maior, evidenciando um vertiginoso crescimento no número de artigos publicados pela China no último quinquênio. Isto se traduz por um crescimento de $426 \%$ da produção da China e apenas $33 \%$ na produção dos Estados Unidos. No mesmo período observa-se um crescimento de $40 \%$ na produção científica brasileira na área.

Uma análise do crescimento da produção científica do Brasil na área é destacada na Figura 2 para as publicações em periódicos indexados em bases de dados consolidadas no período de 1980 a 2016 . O grande crescimento observado indica que o esforço da comunidade acadêmico/científica na produção e divulgação do conhecimento tem contribuído para o avanço da ciência, com reflexos no desenvolvimento econômico, social e cultural do país. A análise deste gráfico mostra que o crescimento da publicação científica em Eletroquímica e Eletroanalítica no Brasil pode ser dividida em várias etapas com velocidades de crescimento representadas por pelo menos três taxas diferentes. Um período de baixa produtividade no intervalo de 1980 a 1989, um aumento expressivo no período de 1990 a 2000 (78\%) e depois um grande aumento entre 2001 a 2011 (151\%) e finalmente uma grande contribuição nos últimos 3 anos.

A avaliação deste desempenho mostra que as atividades de pesquisa e tecnologia caminham concomitantemente com o fortalecimento do Sistema Nacional de Pós-graduação do Brasil. Observa-se no mesmo período igual crescimento na formação de mestres e doutores em nossos Programas de Pós-graduação. Embora a Pós-graduação no Brasil exista desde 1930 (sistema de cátedras) e tenha sido criada

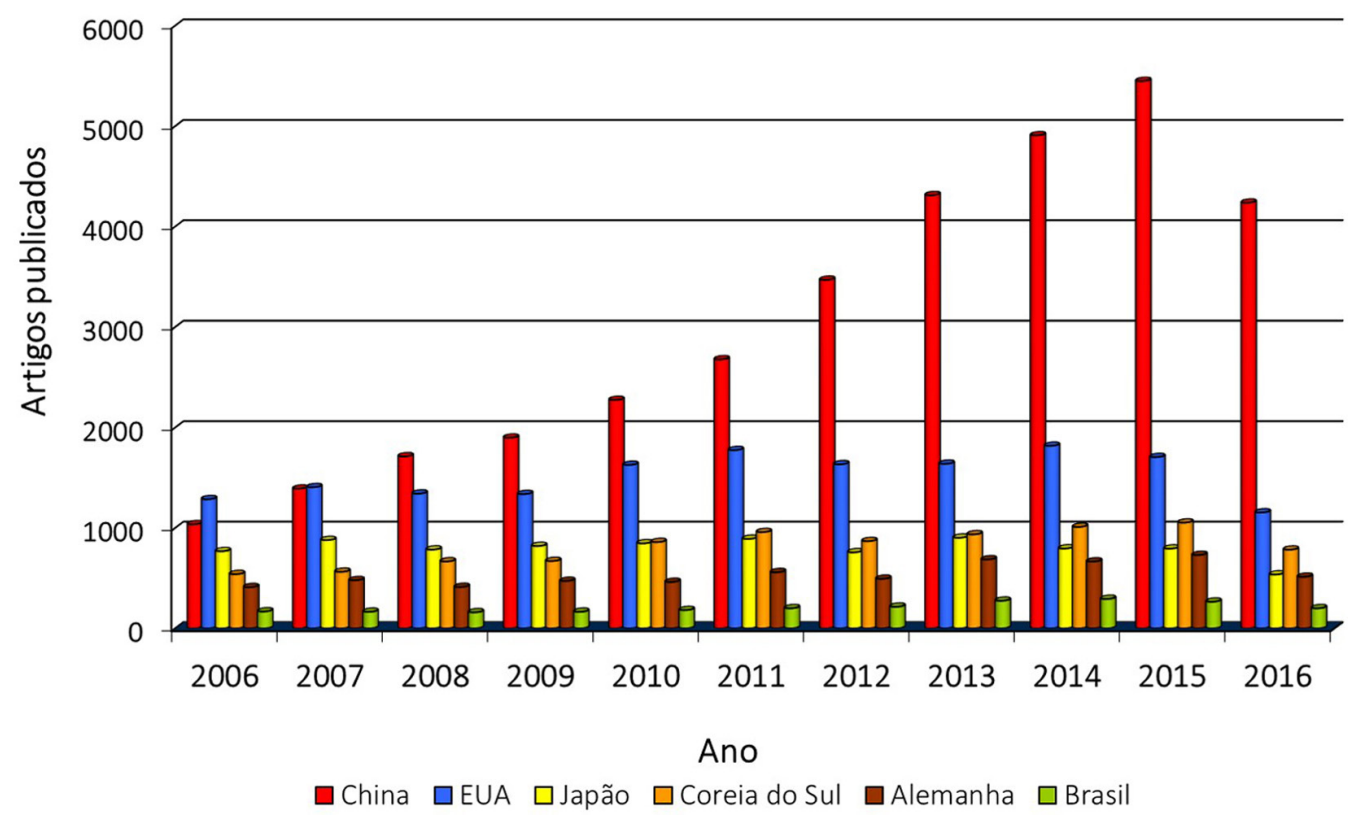

Figura 1. Distribuição das publicações em Eletroquímica e Eletroanalítica selecionadas na base de dados Incites (Thomson Reuters) no período de 2006-2016 para alguns países. Palavra-chave: Electro* 


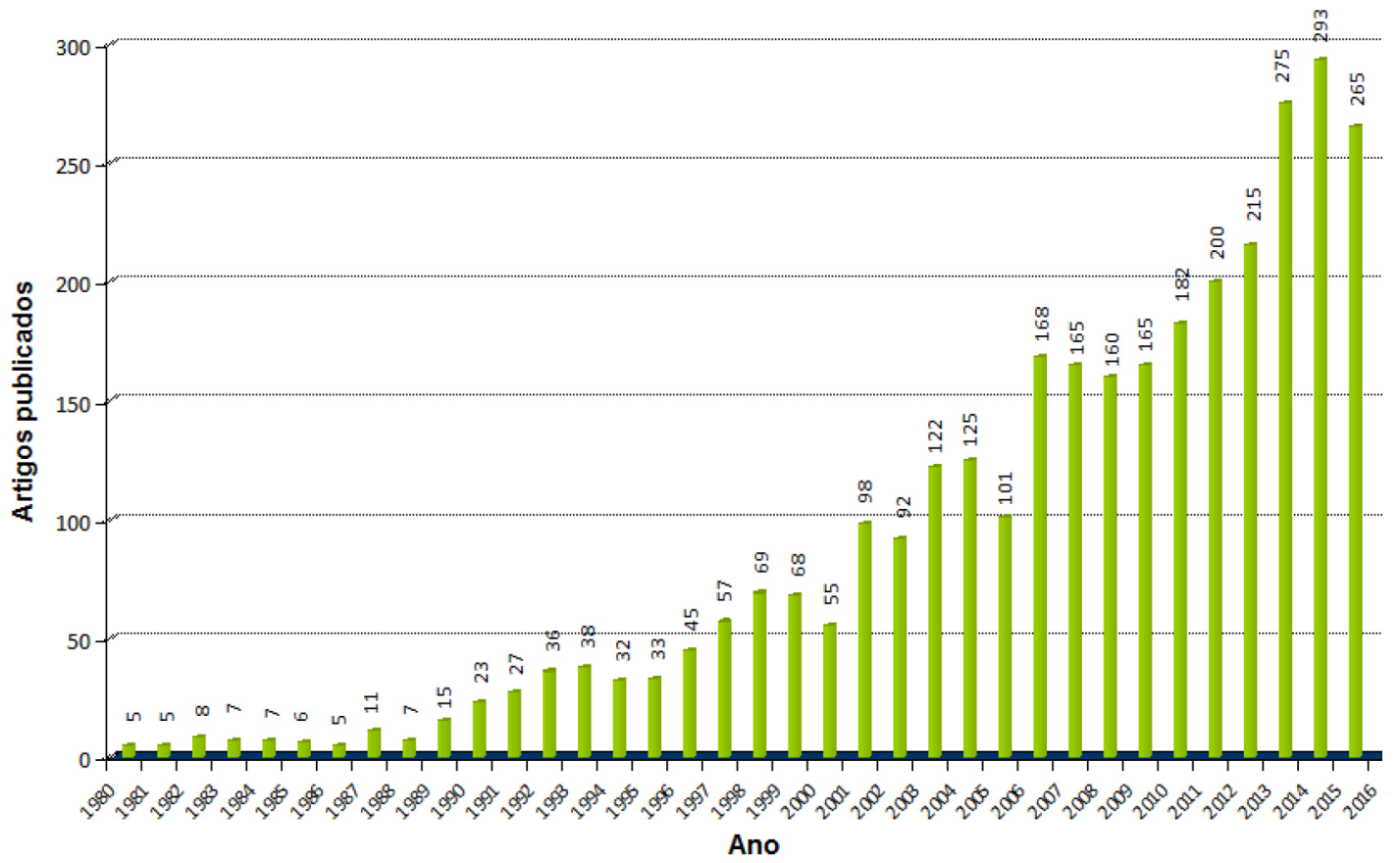

Figura 2. Distribuição das publicações em Eletroquímica e Eletroanalítica selecionadas na base de dados Incites (Thomson Reuters) no período de 1980-2016, Brazil. Palavra-chave: Electro*, Brazil

como Pós-graduação formal em 1965, esta contribuiu para o expressivo crescimento da publicação científica a partir de 1976 com sofisticado sistema de avaliação por indicadores de qualidade, e pelo financiamento dos Programas de Pós-graduação a partir de 2000.

Além disto, deve-se ressaltar a importância das políticas Institucionais envolvendo a formação de redes e grandes projetos nacionais, tais como aqueles induzidos pelo Governo Federal e Governos Estaduais a partir de 1990 (PADCT, fundos setoriais, CEPID, projetos temáticos na FAPESP etc.). Estes resultados mostram que a reunião de pesquisadores com diferentes especialidades de pesquisa em grandes projetos pode fortalecer o compartilhamento e a integração imprescindíveis para uma pesquisa interdisciplinar de qualidade em temáticas da fronteira do conhecimento; estabelecer elos de cooperação com vistas à união interna e inserção externa; melhorar a infraestrutura de pesquisa visando o desenvolvimento de novas ideias e projetos; propiciar avanços na inovação tecnológica em consonância com os anseios da sociedade e estabelecer políticas para ações regulatórias.

A excelência da pesquisa em qualquer área requer condições de infraestrutura adequadas para o desenvolvimento de projetos qualificados e o aporte de recursos das agências de fomento contribuíram sobremaneira para a excelência da infraestrutura em pesquisa a partir de 1990 e formação de recursos humanos qualificados indispensáveis para o profícuo resultado na publicação nos últimos anos.

O impacto e relevância da pesquisa produzida em grupos de pesquisa consolidados só ocorrem após um longo tempo, que em países emergentes envolve a nucleação de ensino de qualidade, titulação do corpo docente, formação de recursos humanos competitivos para enfrentar os desafios com foco nos grandes problemas nacionais, inovação e transferência de conhecimento com abordagens multi, inter- e transdisciplinares de forma a responder aos anseios da sociedade e promover o desenvolvimento com sustentabilidade.

A distribuição da pesquisa produzida em Eletroquímica e Eletroanalítica nos últimos 10 anos nas diversas regiões do país é mostrada na Figura 3, e exemplifica as assimetrias de produtividade na área de acordo com as regiões do país. A região Sudeste é responsável por $69 \%$ da produção científica do Brasil na área de Eletroquímica e Eletroanalítica. Isto pode ser justificado se considerarmos que esta região a foi responsável pela nucleação de grandes grupos de pesquisa atuantes em EE durante muito tempo. Dentre eles destacam-se os grupos consolidados na área na USP/São Paulo, USP/São Carlos, USP/Ribeirão Preto, UFSCar, UNICAMP, UNESP, UFRJ, PUC/ RJ, UFRGS e UFMG que iniciaram e se fortaleceram dentro do contexto do crescimento dos Programas de Pós-graduação dessas Instituições. Atualmente, observa-se que a incorporação de vários pesquisadores formados principalmente nestas Instituições em grupos emergentes de outros estados é responsável, no momento, pela nucleação de novos centros de excelência em pesquisa em EE auxiliados pelo crescimento da Pós-graduação nos estados das outras regiões do país. Políticas indutoras de crescimento científico adotadas pelas agências de fomento $^{1}$ (ex: $30 \%$ dos auxílios a pesquisa são destinados a regiões NE, CO e N programas de bolsas e parcerias entre programas emergentes e consolidados fomentados pela CAPES) têm auxiliado

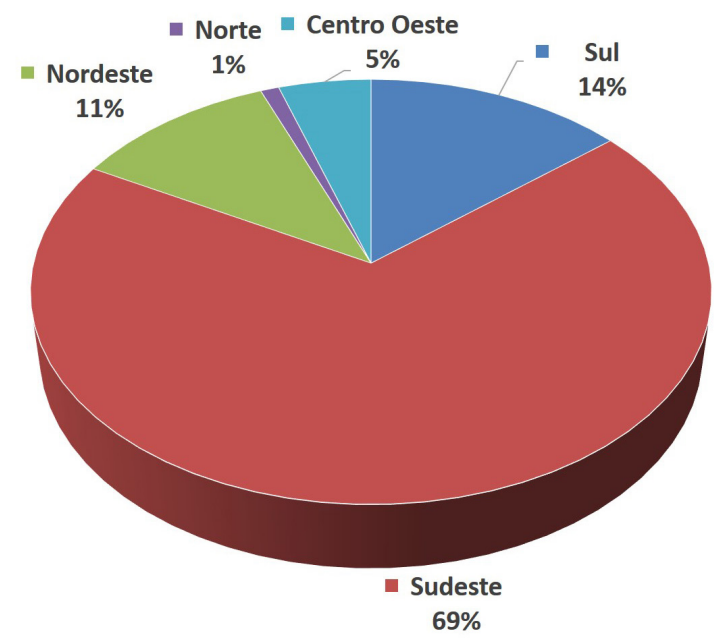

Figura 3. Contribuição das publicações em Eletroquímica e Eletroanalítica selecionadas na base de dados Incites (Thomson Reuters) no período de 20062015 de acordo com as regiões do Brasil. Palavra chave: Electro*, Brazil 
sobremaneira o desenvolvimento destes novos grupos de pesquisa nas regiões a corrigir esta assimetria e acelerar o crescimento da produção científica, traduzido pelo exponencial aumento nos últimos 5 anos.

De acordo com dados publicados recentemente por Cândido et al., ${ }^{2}$ outro dado interessante refere-se à distribuição de bolsistas de produtividade em pesquisa divulgados pelo $\mathrm{CNPq}$ na área de Química. As especialidades de Eletroquímica e Eletroanalítica se inserem nas subáreas de Físico-Química (FQ) e Química Analítica (QA), respectivamente. Neste contexto, no período de 2003-2013 a Eletroquímica contou com 32 bolsistas de produtividade $(18 \%$ dos bolsistas da subárea de FQ). O número de artigos publicados no período (dados do CV Lattes) por estes pesquisadores foi de 1603 e representa $18 \%$ da produção dos pesquisadores (8144) da subárea de Físico-Química. A qualidade destas publicações pode ser constatada pela média das citações/artigo que atinge 21,57 e fator de impacto médio de 3,03, média superior à área de química que é 16,14 e fator de impacto médio de 2,55. Do mesmo modo, a Eletroanalítica se destaca na subárea de Química Analítica, com 21 pesquisadores representando $12 \%$ na subárea e publicação de 1189 artigos no período de 2003-2013 (15\% da produção científica na QA). A média de citações é de 15,76 e fator de impacto médio de 2,76. Índices menores apenas da especialidade de Instrumentação Analítica dentro da subárea de QA. Considerando o fator de impacto (FI) dos periódicos onde são publicados os artigos observa-se que a maioria dos artigos foi divulgado em periódicos com $3<\mathrm{FI}<4$. Dentre os 208 periódicos usados na busca na base de dados "InCites", observa-se que as 10 revistas científicas com maior número de publicações da comunidade de EE Brasileira são: Journal of the Electrochemical Society, Sensors and Actuators B-Chemical, Electroanalysis, Electrochimica Acta, Journal of Electroanalytical Chemistry, Electrochemistry Communications, Journal of Power Sources, Biosensors \& Bioelectronics, International Journal of Hydrogen Energy and Sensors.

Um aspecto importante que expressa o grau de maturidade científica em uma determinada área é a formação das sociedades científicas, que auxiliam sobremaneira na ampliação do contato e o conhecimento entre os pesquisadores atuantes em áreas afins, bem como na tomada de decisões conjuntas capazes de melhor nortear, oxigenar e favorecer o rápido progresso científico. No Brasil, a fundação da SBQ em 1977 foi a responsável pelo desenvolvimento e crescimento da química em todo o Brasil. No entanto, a Divisão de Eletroquímica e Eletroanalítica no Brasil foi criada apenas em 1990 e implantada em 1993. Esta divisão conta em média ao longo dos anos com 110 sócios efetivos que têm se reunido anualmente junto com convidados internacionais para discutir os avanços e as tendências da pesquisa em Eletroquímica e Eletroanalítica como ciência inovadora e inserida na resolução dos grandes problemas nacionais. No entanto, os trabalhos de EE, desde 1978, são apresentados nos Simpósios Brasileiros de Eletroquímica e Eletroanalítica (SIBEE), que tem se constituído em um respeitável fórum de discussão, ${ }^{3}$ como demonstrado pelo crescimento dos trabalhos apresentados no Simpósio a cada ano (Figura 4). O expressivo número de trabalhos científicos sempre primou pela qualidade e em 2013-2015 foi criada a SBEE (Sociedade Brasileira de Eletroquímica e Eletroanalítica) do Brasil, efetivada em 2016.

Como discutido preliminarmente, devido à natureza multidisciplinar, a Eletroquímica e Eletroanalítica ainda estão representadas nas reuniões bianuais do ENQA, ABRACO (Associação Brasileira de Corrosão), INTERCORR - evento internacional de corrosão realizado no Brasil, EBRATES (Encontro Brasileiro de Tratamento de Superfícies), ENBAT (Encontro Nacional de Produtores de Baterias Chumbo-Ácido), CBECIMAT (Congresso Brasileiro de Engenharia e Ciência dos Materiais). A eletroquímica também aparece em menor grau em outros congressos e encontros como Congresso Brasileiro de Cerâmica, Congresso Brasileiro de Energia, Congresso Brasileiro de Catálise e outros de outras áreas do conhecimento.

Deste modo, a análise quantitativa da Eletroquímica e Eletroanalítica no Brasil aponta indicadores extremamente favoráveis. Uma análise qualitativa desta produção indicando as tendências e desafios dentro de cada especialidade é apresentada a seguir.

\section{AVANÇOS E TENDÊNCIAS DA ELETROQUÍMICA E ELETROANALÍTICA NO BRASIL}

\section{Eletroquímica}

A Eletroquímica é uma área da ciência bastante ampla e está incorporada em vários ramos da química, assim os campos de interesse da eletroquímica atual no Brasil podem ser, em parte, delineados com base na distribuição dos trabalhos dos SIBEEs nos últimos 6 anos. Nestes SIBEEs, os trabalhos foram distribuídos nas áreas: bioeletroquímica e biocélulas a combustível, ${ }^{4}$ corrosão, ${ }^{5-7}$ eletrocatálise,${ }^{8,9}$ eletroquímica ambiental, ${ }^{10}$ eletroquímica orgânica, ${ }^{11}$ energia,,${ }^{12,13}$ materiais nanoestruturados, ${ }^{14}$ passivação ${ }^{15}$ e tratamento de superfícies. ${ }^{16}$ Essa divisão foi colocada em ordem alfabética e não reflete a importância/abundância de pesquisadores em uma área específica. Dada a sua interdisciplinaridade,

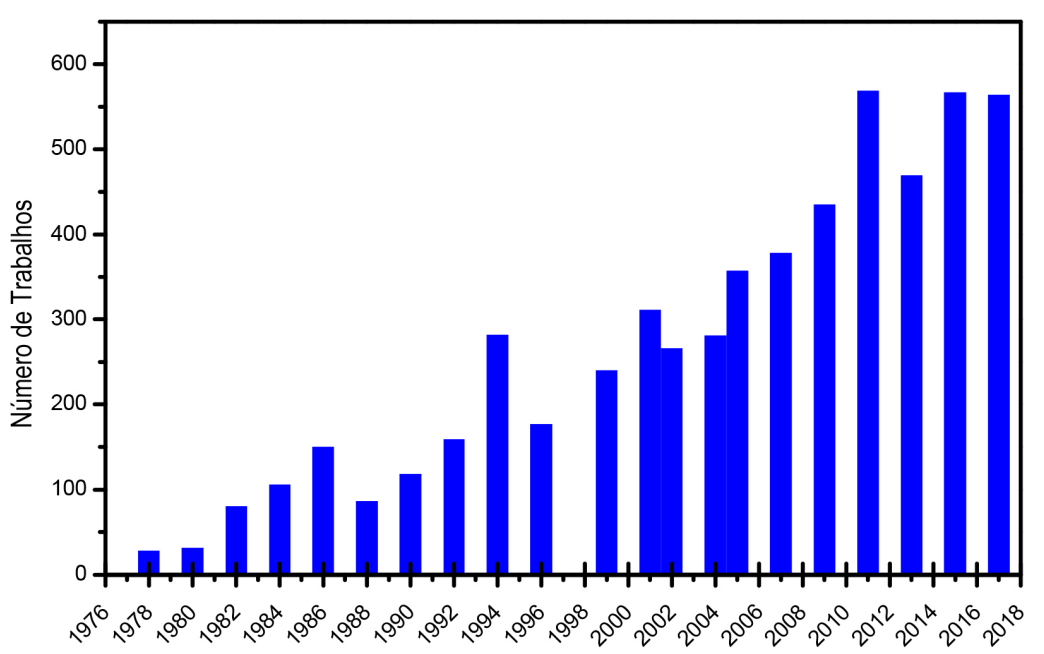

(Ano) 
muitas vezes, um mesmo grupo de pesquisa atua em mais de uma das áreas especificadas. O SIBEE e mais tarde a Divisão de Eletroquímica e Eletroanalítica da SBQ desempenharam papel importante na nucleação e na disseminação da Eletroquímica/Eletroanalítica no Brasil, os grupos se multiplicaram, principalmente, no interior do Estado de São Paulo e em outros Estados e regiões do País.

A Eletroquímica brasileira tem acompanhado a tendência da Eletroquímica mundial, inicialmente com estudos fundamentais de cinética eletroquímica e mecanismos de reação de compostos químicos orgânicos e inorgânicos, organometálicos e compostos de coordenação. Pouco a pouco a área foi se movendo para estudos de materiais, tais como corrosão e proteção, eletrocatálise, eletrólise, síntese eletroquímica, tratamento de águas residuárias, energia e áreas de interface tais como fotoeletroquímica, bioeletroquímica, splitting da água, obtenção eletroquímica de nanofios, nanobastões magnéticos ou não, materiais nanoestruturados. A Figura 5 resume os resultados de uma busca bibliográfica realizada na base de dados do Web of Science de algumas subáreas selecionadas que têm publicações de brasileiros.

Quando se fala no futuro desta área, deve-se ter em mente os grandes desafios da atualidade e como os eletroquímicos poderão atuar na proposição de soluções e inovações.

O entendimento da eletrocatálise envolve estudos com eletrodos de metais nobres; eletrodos de óxidos/hidróxidos; ligas metálicas na ausência de metais nobres; ligas leves; intermetálicos; nanomateriais e biomateriais utilizados em processos de produção/armazenamento de energia para células a combustível, baterias, capacitores, supercapacitores, splitting da água para produção limpa de hidrogênio etc. Nesse campo o maior desafio é entender, em nível atômico/ molecular, o processo da transferência eletrônica e a cinética global dos processos envolvidos.

A proteção de materiais contra corrosão tem desafios específicos na busca de revestimentos metálicos de metais ativos ( $\mathrm{Al}, \mathrm{Cu}, \mathrm{Zn}$ e outros); metálico-cerâmicos; revestimentos poliméricos reforçados com nanopartículas, grafeno, óxido de grafeno etc. A fragilização por hidrogênio tem merecido atenção, porém, a determinação eletroquímica da quantidade de hidrogênio que ingressa em ligas de alumínio e na região de solda continua sendo um desafio para a área.

O desenho de moléculas, catalisadores, materiais inteligentes, biomateriais etc., que apresentem funções específicas como, por exemplo, inibidores de corrosão, transporte e liberação controlada de medicamentos, modificações de superfícies e outras constitui um grande desafio para os eletroquímicos, químicos, engenheiros e físicos teóricos.
O estudo da corrosão atmosférica e de inibidores voláteis de corrosão (VCI) ainda se constitui um grande desafio para os eletroquímicos quando se deseja comparar dados eletroquímicos de laboratório com dados obtidos a partir de ensaios de campo e ensaios em câmara úmida.

A eletrodeposição de metais e ligas metálicas magnéticas ou não vem sendo dirigida para a obtenção de materiais em líquidos iônicos e líquidos análogos, preparação de nanofios, nanobastões magnéticos ou com propriedades tais que possam ser utilizados no splitting da água, fabricação de sensores etc.

A Eletroquímica brasileira é ativa também no estudo de minerais, especialmente sulfetos metálicos, buscando recuperar metais com alto valor agregado e reduzir rejeitos da indústria de mineração. Neste caso, há um imenso desafio imposto à eletroquímica na busca por metodologias que acelerem a lixiviação e biolixiviação da calcopirita.

Em nosso ponto de vista, a Eletroquímica no Brasil ainda está distante dos mais variados processos de produção industrial de compostos químicos, dos processos de pintura eletroforética empregados na indústria, de uma contribuição direta para a produção e armazenamento de energia, da produção de fármacos e muitos outros. A interação efetiva com a indústria vai exigir mais do que conhecimentos sólidos de Eletroquímica/Química, provavelmente, exigirá uma mudança de comportamento com complementação da formação dos pesquisadores para atuarem conjuntamente em nichos de atividades industriais onde a Eletroquímica se faz necessária. Verifica-se, no entanto, que há vários grupos exercendo ou se capacitando para esse desafio.

$\mathrm{Na}$ opinião dos autores, esses são alguns dos desafios que os eletroquímicos brasileiros terão que vencer nos próximos anos, no entanto, cada membro da comunidade eletroquímica poderia acrescentar outros.

As várias Sociedades Científicas e Fundações de fomentos devem gerar novas estratégias capazes de disseminar e fortalecer núcleos de pesquisas em eletroquímica para trazer soluções de problemas da indústria e sociedade brasileiras.

\section{Eletroanalítica}

Os grandes avanços na área de Eletroanalítica ocorreram sempre nas fronteiras do conhecimento em química analítica, novos materiais, novas instrumentações, tratamento de dados (quimiometria), fundamentos da eletroquímica e aplicações diretas na área de alimentos, ambiente, saúde, energia e indústria. Pelo seu caráter inter e transdisciplinar tem sido suporte para o desenvolvimento de diversas

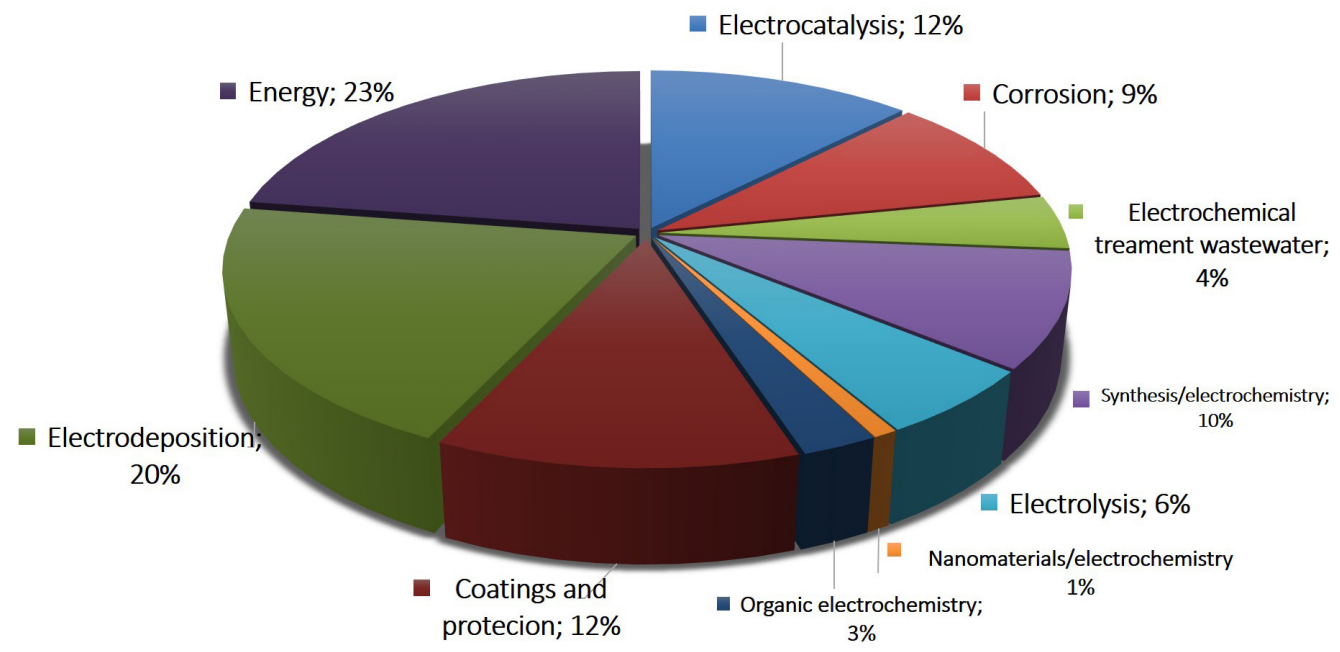

Figura 5. Distribuição das Publicações em Eletroquímica no Brasil selecionadas na base de dados Web of Science (Thomson Reuters) no período de $2002-2017$ 
áreas da química e órgãos de fiscalização, validação e controle de produtos e processos. Isto dificulta sobremaneira uma avaliação da área em termos quantitativos, pois há uma grande diversificação das publicações em periódicos relevantes em áreas correlatas, difíceis de serem avaliados dentro de um contexto específico.

A Eletroanalítica no Brasil foi implementada a partir de 1952 na USP, após o pós-doutorado do Prof. P. Senise na área de Eletroanálise nos EUA, ${ }^{17-20}$ que começou uma profícua escola na área, formando recursos humanos altamente qualificados que se espalharam pelo país durante várias gerações consecutivas. Hoje a Eletroanalítica possui grupos de pesquisa representativos em todo o país. ${ }^{17-21}$

Dentro deste contexto, o futuro da Eletroanalítica como especialidade da área de Química Analítica foi destacado previamente em excelentes publicações sobre a pesquisa em Química Analítica no Brasil por diversos pesquisadores brasileiros. ${ }^{19,20}$ Dentre os inúmeros indicadores que contribuíram para os avanços da eletroanalítica deve-se considerar o melhoramento na instrumentação eletroanalítica e o melhor entendimento dos processos eletródicos. ${ }^{22,23} \mathrm{O}$ desenvolvimento das técnicas voltamétricas de pulso e onda quadrada e as técnicas de redissolução aumentaram a competitividade das técnicas eletroanalíticas ampliando os limites de detecção para um grande número de analitos. Nos anos mais recentes, grandes avanços têm sido alcançados quanto ao nível de detecção usando as técnicas potenciométricas e novos materiais têm sido desenvolvidos. ${ }^{24} \mathrm{Uma}$ das maiores aplicações das técnicas condutométricas tem sido nas determinações usando cromatografia iônica e detecção em processos cromatográficos. ${ }^{21}$ Ambas possuem destaque devido à facilidade de resposta e ampla aplicabilidade do método, no entanto, a literatura tem reportado mais metodologias eletroanalíticas baseadas em métodos voltamétricos e mais recentemente envolvendo a espectroscopia de impedância eletroquímica, capacitância e imitância eletroquímica. ${ }^{25}$ A possibilidade de miniaturização dos eletrodos (microeletrodos, eletrodos impressos descartáveis) foi importante na diminuição da dimensão das células eletroquímicas e na redução do volume do analito, ampliando sua aplicabilidade ao entendimento dos sistemas "in vivo". ${ }^{26,27}$ Desde a década de 1980 o Brasil vem desenvolvendo importantes contribuições na área de eletrodos modificados com vistas ao desenvolvimento de sensores eletroquímicos e tem sido revisada na literatura. ${ }^{28-31}$ Neste contexto, um dos grandes avanços da área consiste na modificação da superfície do eletrodo, o que abriu inúmeras possibilidades para desenvolvimento de sensores e biossensores, contribuindo de modo singular para o avanço da eletroanalítica moderna. ${ }^{32}$ A versatilidade dos modificadores (ligantes, polímeros, óxidos, orgânicos, sílicas, metais, complexos, compostos biológicos, enzimas, DNA, proteínas, anticorpos, antígenos, organismos, tecidos, células, organelas, membranas etc.) abriu grande possibilidade de desenvolver métodos analíticos mais rápidos, sensíveis, precisos e seletivos e, principalmente, de baixo custo.

Neste contexto, nos últimos anos tem-se observado o desenvolvimento de eletrodos bioinspirados, ${ }^{33}$ monocamadas autorganizadas, ${ }^{34}$ microfluídica, ${ }^{35}$ e point-of-care test (POCT). ${ }^{36} \mathrm{O}$ emprego de nanopartículas magnéticas para pré-concentrar o analito de amostras complexas e o emprego da eletroanalítica em papel são alguns exemplos. ${ }^{37}$ Um desafio importante para o desenvolvimento dos sensores seria investigar receptores que mantenham a atividade por longo tempo. Outro desafio é encontrar biomarcadores em amostra de saliva para o diagnóstico de doenças, o que facilitaria a amostragem quando comparada com sangue. ${ }^{38}$ Mais recentemente, tem-se observado a modificação de eletrodos com materiais biomiméticos usando a tecnologia de impressão molecular, ${ }^{39}$ nas quais se incluem os polímeros impressos molecularmente (molecularly imprinting polymers, MIP) que tem aumentado o reconhecimento seletivo de diversos analitos. Uma das áreas de destaque na utilização de eletrodos modificados com diferentes materiais, tais como, polímeros quimicamente impressos, nanotubos de carbono, grafeno e nanopartículas metálicas, assim como a utilização de técnicas eletroquímicas amperométricas e o desenvolvimento das línguas eletrônicas. As línguas eletrônicas fazem parte da evolução tecnológica para a detecção de diversas substâncias ou produtos em tempos relativamente pequenos. ${ }^{40} \mathrm{O}$ acoplamento de eletrodos modificados às técnicas de separação tem proporcionado a determinação de vários compostos simultaneamente em amostras diversas, ampliando os limites de detecção, aumentando a sensibilidade analítica e proporcionando medidas com maior precisão. O desenvolvimento destes detectores tem sido proporcionado pelos inúmeros avanços na modificação de superfícies eletródicas como, por exemplo, nanotubos de carbono, grafeno e nanopartículas metálicas. Adicionalmente, outros desenvolvimentos têm contribuído para melhorar a detecção eletroquímica nas técnicas CLAE como, por exemplo, a utilização de líquidos iônicos como eluente e a utilização de técnicas de geração de pulsos diferenciados. ${ }^{41,42}$

Adicionalmente, outra grande contribuição para a detecção eletroquímica tem sido a utilização de sensores eletroquímicos que utilizam a técnica de condutimetria acoplada à técnica de eletroforese capilar. Esta contribuição tem permitido a detecção de espécies com pequenos limites de detecção, bem como aberto uma nova possibilidade de detecção eletroquímica condutométrica sem o contato físico da solução com os eletrodos. ${ }^{35}$

Deste modo, embora seja difícil citar todas as relevantes contribuições dos grupos de Eletroanalítica do Brasil, é possível concluir que a comunidade científica tem feito grandes progressos em eletroanálise tanto em micro como em nanoescala, no desenvolvimento de novos sensores e dispositivos competitivos com a determinação de analitos de interesse biológico e ambiental em níveis compatíveis com técnicas pouco econômicas e que demandam grande tempo de análise.

\section{CONCLUSÃO}

A análise crítica dos indicadores de pesquisa apresentados neste artigo mostra que a publicação científica nas áreas de Eletroquímica e Eletroanalítica no país aumentou exponencialmente nos últimos 10 anos (2006-2015) e o Brasil é líder na América Latina nestas áreas. Outrossim, observa-se também que a qualidade desta produção pode ser aferida pelo alto impacto destas publicações e na formação de recursos humanos altamente qualificados. A ampliação dos Programas de Pós-graduação em Química e o consequente aumento expressivo no número de grupos de pesquisa emergentes em todos os Estados do país têm contribuído para a correção das assimetrias observadas no passado, onde os grandes pilares da Eletroquímica e Eletroanalítica estavam alicerçados no Estado de São Paulo. Dentro deste contexto, a ampliação dos quadros da EE no país ainda se deve ao baixo custo da instrumentação requerida em relação a outras áreas, ao incentivo à formação de redes de excelência em pesquisa e aos grandes projetos de cooperação que permitem o compartilhamento de ideias, tecnologias, infraestrutura, equipamentos e o fortalecimento dos cursos de pós-graduação, consolidados e em consolidação, com reflexos diretos no avanço da qualidade da ciência produzida em todas as áreas.

\section{AGRADECIMENTOS}

Os autores agradecem a Orlando Fatibello Filho pela revisão do texto e ao financiamento da FAPESP, CNPq e CAPES.

\section{REFERÊNCIAS}

1. Barral-Neto, M.; de Felício, J. R. D.; Camargo, E. P. O.; Revista USP, 2007, 73, 48 . 
2. Cândido, L. F. O.; Santos, N. C. F.; Rocha, J. B. T. da; Quim. Nova 2016, 39, 393.

3. Avaca, L. A.; Tokoro, R.; Quim. Nova 2002, 25, 25.

4. Rasmussen, M.; Abdellaoui, S.; Minteer, S. D.; Biosens. Bioelectron. 2016, 76, 91.

5. Pokorný, P.; Tej, P.; Kou il, M.; Constr. Build. Mater. 2017, 132, 271.

6. Jamali, S. S.; Mills, D. J.; Prog. Org. Coatings 2016, 95, 26.

6. Polcari, D.; Dauphin-Ducharme, P.; Mauzeroll, J.; Chem. Rev. 2016, 116, 13234.

8. Stamenkovic, V. R.; Strmcnik, D.; Lopes, P. P.; Markovic, N. M.; Nat. Mater. 2017, 16, 57.

9. Suen, N.-T.; Hung, S.-F.; Quan, Q.; Zhang, N.; Xu, Y.-J.; Chen, H. M.; Jeong, H. Y.; Joo, S. H.; Zhang, Y.; Wood, D.; Zelenay, P.; More, K.; Stroh, K.; Zawodzinski, T.; Boncella, J.; McGrath, J. E.; Inaba, M.; Miyatake, K.; Hori, M.; Ota, K.; Ogumi, Z.; Miyata, S.; Nishikata, A.; Siroma, Z.; Uchimoto, Y.; Yasuda, K.; Kimijima, K. -i.; Iwashita, N.; Chem. Soc. Rev. 2017, 46, 337.

10. Zhang, S.; Zhang, Z.; Zhao, R.; Gu, J.; Liu, J.; Örmeci, B.; Zhang, J.; Chem. Eng. Commun. 2017, 204, 265.

11. Genovino, J.; Sames, D.; Hamann, L. G.; Touré, B. B.; Angew. Chemie Int. Ed. 2016, 55, 14218.

12. An, L.; Zhao, T. S.; J. Power Sources 2017, 341, 199.

13. Canepa, P.; Sai Gautam, G.; Hannah, D. C.; Malik, R.; Liu, M.; Gallagher, K. G.; Persson, K. A.; Ceder, G.; Chem. Rev. 2017, 117, 4287.

14. Share, K.; Westover, A.; Li, M.; Pint, C. L.; Chem. Eng. Sci. 2016, 154, 3.

15. Veluchamy, A.; Sherwood, D.; Emmanuel, B.; Cole, I. S.; J. Electroanal. Chem. 2017, 785, 196.

16. Wen, Q.; Guo, Z.; Chem. Lett. 2016, 45, 1134.

17. Gutz, I. G. R.; Quim. Nova 2006, 29, 1150.

18. Interview of Orlando Fatibello Filho; Br. J. Anal. Chem., 2013, 3, pag. X. http://www.brjac.com.br/, acessada em Março 2017.

19. Ferreira, S. L. C.; Pereira, P. A. de P.; Nóbrega, J. A.; Fatibello-Filho, O.; Feres, M. A.; Reis, B. F.; Bruns, R. E.; Aquino Neto, F. R. de; Anal. Lett. 2008, 41, 1494.
20. de Andrade, J.; Korn, M. G.; Cadore, S.; Microchem. J. 2004, 77, 101.

21. Stradiotto, N. R.; Yamanaka, H.; Zanoni, M. V. B.; J. Braz. Chem. Soc. 2003, 14, 159.

22. Bond, A. M.; Anal. Chim. Acta 1999, 400, 333.

23. Brett, C. M. A.; Electroanalysis 1999, 11, 1013.

24. Torres, K. Y. C.; Marzal, P. C.; Kubota, L. T.; Bakker, E.; Quim. Nova 2006, 29, 1094.

25. Fernandes, F. C. B.; Patil, A. V.; Bueno, P. R.; Davis, J. J.; Anal. Chem. 2015, 87, 12137.

26. Wightman, R. M.; Wipf, D. O.; Acc. Chem. Res. 1990, 23, 64.

27. Nascimento, V. B.; Angnes, L.; Quim. Nova 1998, 21, 614.

28. Vidotti, M.; Torresi, R.; Torresi, S. I. C. de; Quim. Nova 2010, 33, 2176.

29. Lowinsohn, D.; Bertotti, M.; Quim. Nova 2006, 29, 1318.

30. Freire, R. S.; Pessoa, C. A.; Kubota, L. T.; Quim. Nova 2003, 26, 381.

31. Souza, M. de F. B.; Quim. Nova 1997, 20, 191.

32. Arduini, F.; Cinti, S.; Scognamiglio, V.; Moscone, D.; Palleschi, G.; Anal. Chim. Acta 2017, 959, 15.

33. Zapp, E.; Souza, F. D.; Souza, B. S.; Nome, F.; Neves, A.; Vieira, I. C.; Bunton, C. A.; Nome, F.; Neves, A.; Gahan, L. R.; Hanson, G. R.; Riley, M. J.; Schenk, G. A.; Gahan, L. R.; Analyst 2013, 138, 509.

34. Coelho, D.; Machado, S. A. S.; Electrochim. Acta 2014, 142, 191.

35. Coltro, W. K. T.; Lima, R. S.; Segato, T. P.; Carrilho, E.; de Jesus, D. P.; do Lago, C. L.; da Silva, J. A. F.; Breadmore, M. C.; Vellekoop, M. J.; Bossche, A.; Dedem, G. W. K. van; Anal. Methods 2012, 4, 25.

36. Moreira, F. T. C.; Dutra, R. A. F.; Noronha, J. P. C.; Fernandes, J. C. S.; Sales, M. G. F.; Sensors Actuators B Chem. 2013, 182, 733.

37. Nery, E. W.; Kubota, L. T.; Anal. Chim. Acta 2016, 918, 60.

38. Campuzano, S.; Yánez-Sedeño, P.; Pingarrón, J. M.; TrAC Trends Anal. Chem. 2017, 86, 14

39. Tarley, C. R. T.; Sotomayor, M. D. P. T.; Kubota, L. T.; Quim. Nova 2005, 28, 1087.

40. Tahara, Y.; Toko, K.; IEEE Sens. J. 2013, 13, 3001.

41. Honeychurch, K.; Separations 2016, 3, 28.

42. Trojanowicz, M.; Anal. Chim. Acta 2011, 688, 8. 